\title{
Supplementary Materials for Estimating a Parametric Component Lifetime Distribution from a Collection of Superimposed Renewal Processes
}

\author{
Wei Zhang \\ Department of Statistics \\ Iowa State University \\ Ames, IA 50011 \\ wzhang87@iastate.edu \\ Luis A. Escobar \\ Department of Experimental Statistics \\ Louisiana State University \\ Baton Rouge, LA 70808 \\ luis@lsu.edu
}

\author{
Ye Tian \\ Department of Statistics \\ Iowa State University \\ Ames, IA 50011 \\ tianye1984@gmail.com \\ William Q. Meeker \\ Department of Statistics \\ Iowa State University \\ Ames, IA 50011 \\ wqmeeker@iastate.edu
}

\section{Overview}

We proposed a likelihood-based method for estimating the lifetime distribution of a system component from the aggregated event data consisting of superpositions of renewal processes (SRP). In these supplementary materials, we present the detailed results of the simulation study that we used to evaluate the performance of the proposed incomplete-data ML (idML) estimator. We also present the results of another simulation study to better understand the partition probabilities and how to use them to explain the behavior of the relative efficiency of the idML estimator. 


\section{Simulation Results}

This section describes a simulation study that investigates the efficiency of the idML estimator relative to the complete-data ML (cdML) estimator, and compares two interval estimation methods based on Wald approximation and the likelihood ratio test.

\subsection{Summary of the simulation design}

In the simulation study, we assume the components in each system follow a Weibull lifetime distribution with scale parameter $\eta=1$, and the number of socket $m$ is the same for all systems in the fleet. The factors used in the simulation study include the number of sockets $m$, the number of events per system $r$, the number of events in the fleet $n \times r$ and the Weibull shape parameter $\beta$. We carefully controlled the levels of the factors and studied their effects on the idML estimator. The details of the simulation design are described in the original paper.

\subsection{Variance and bias of the idML estimator}

Figures 1 and 2 present the results of 50 Weibull idML and cdML estimates randomly chosen from the total 5,000 simulations for two of the factor level combinations. Note that the longer, thicker line in each plot corresponds to the true Weibull distribution $(\eta=1$ and $\beta=3)$.
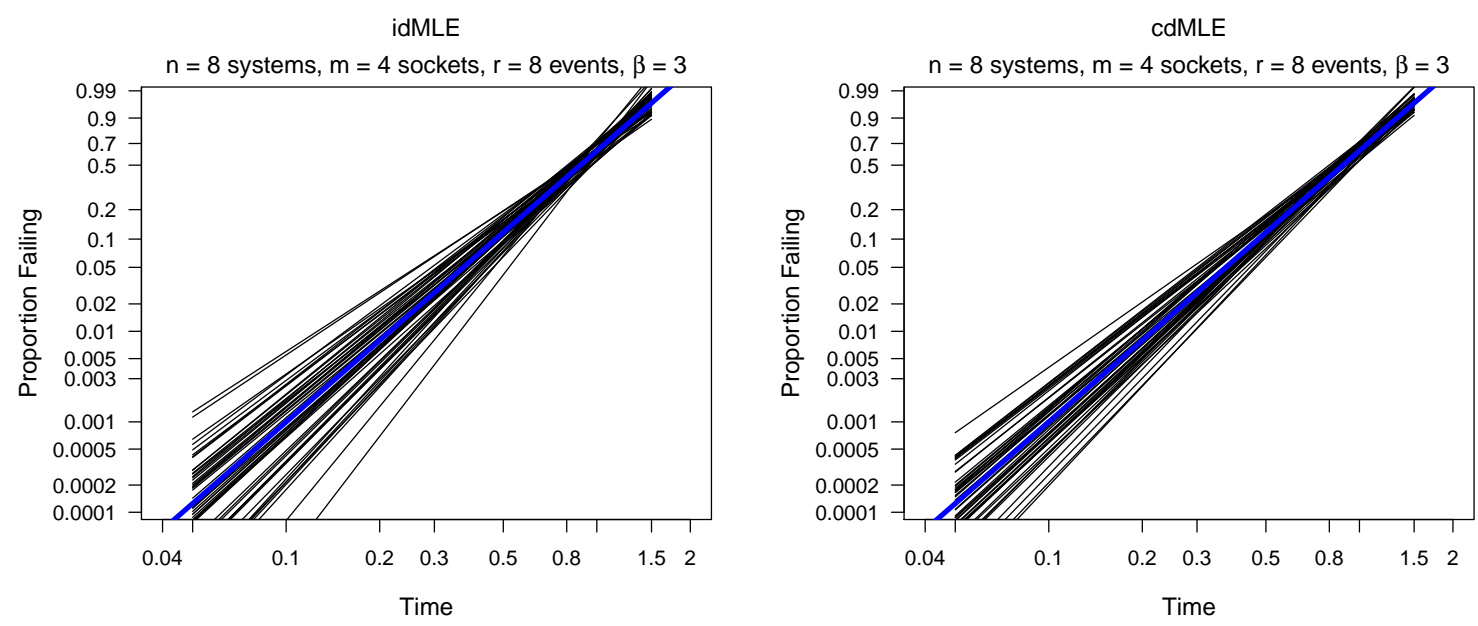

Figure 1: Summary showing the idML estimates (left) and the cdML estimates (right) for the 50 simulated data sets for Case 1 where $n=8, m=4, r=8$.

Figure 1 corresponds to Case 1 where the fleet has $n=8$ systems, each system has $m=4$ sockets and the number of events per system is $r=8$. The two plots compare the idML estimates (the left plot) with the cdML estimates (the right plot) for the same 50 data sets that are randomly selected from the total 5,000 simulated data sets for case 1. As shown in the plots, the idML estimator has a variance that is relatively larger than that of the cdML estimator. 

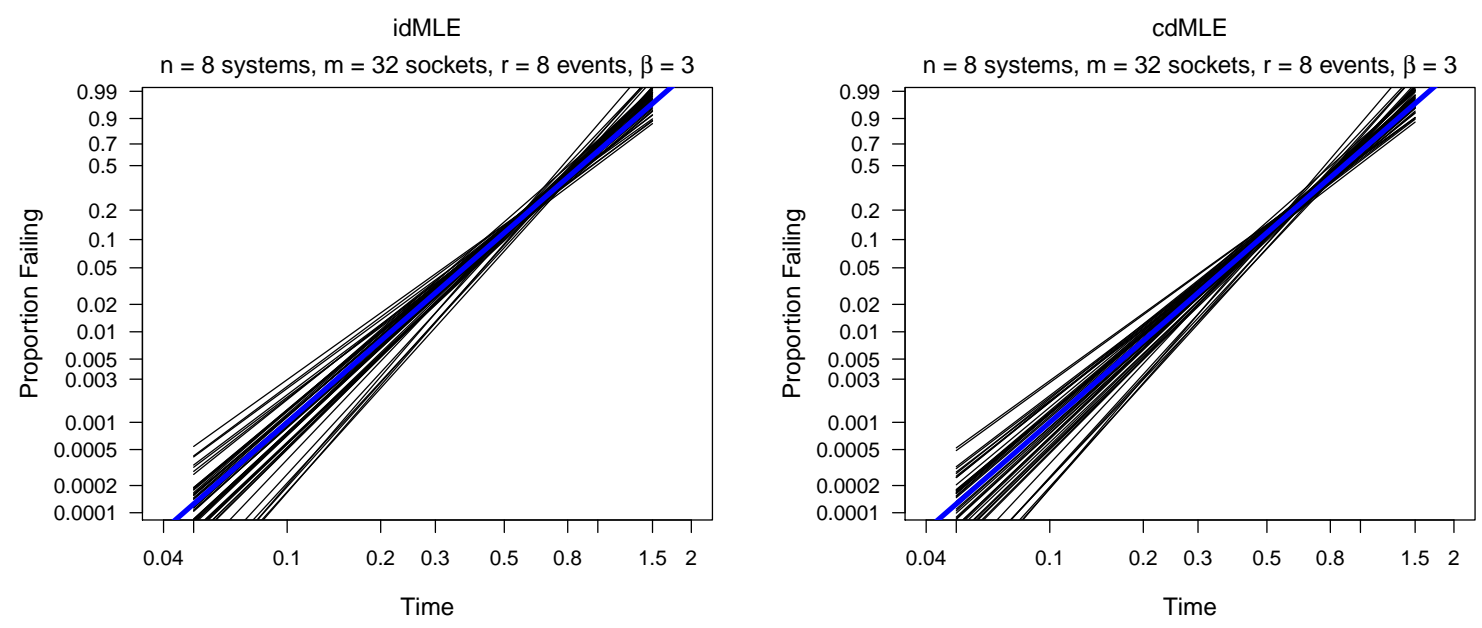

Figure 2: Summary showing the idML estimates (left) and the cdML estimates (right) for the 50 simulated data sets for Case 2 where $n=8, m=32, r=8$.

Figure 2 refers to Case 2 where $n=8, m=32$, and $r=8$. In this case the idML and cdML estimators have comparable performances in the sense that their estimates have similar spread around the true distribution.

\subsection{Relative efficiency of the idML estimator}

For each parameter of interest, we calculate the estimate of the MSE based on the 5,000 simulated data sets. Then the ratio of the respective MSE estimates gives the relative efficiency (RE) of the idML estimator relative to the cdML estimator.

\section{The number of events $(r \times n)$ effect}

First, we keep the number of sockets $m$ within each system fixed and vary the number of events $r$ in each system, the number of events in the fleet of systems $(r \times n)$, and the Weibull shape parameter $\beta$. Figure 3 shows a set of relative efficiency curves for three different values of $r(2$, 4 , and 8 ) and two different values of $\beta$ (1 and 3), keeping the number of sockets in each system fixed at $m=16$. The top-left plot in Figure 3 shows the RE curves of the idML estimator for estimating the Weibull shape parameter $\beta=1$. Within each system, the number of sockets $m$ is fixed at 16. The curves correspond to values of $r=2,4$, and 8 . The $x$-axis in the plot represents the total number of events in the whole fleet $(r \times n)$. For example, suppose $r=4$, when the number of systems $n$ is set at values $2,4,8,16,32$, and 64 , the total number of events in the fleet would be $8,16,32,64,128$, and 256 , respectively. The $y$-axis shows the RE for the Weibull shape parameter $\beta$. Similarly, the top-right plot in Figure 3 shows the RE for Weibull shape parameter $\beta$ with $\beta=3$. The bottom-left and bottom-right plots in Figure 3 present the RE for estimating the quantile $t_{0.1}$ with $\beta=1$ and $\beta=3$, respectively. 

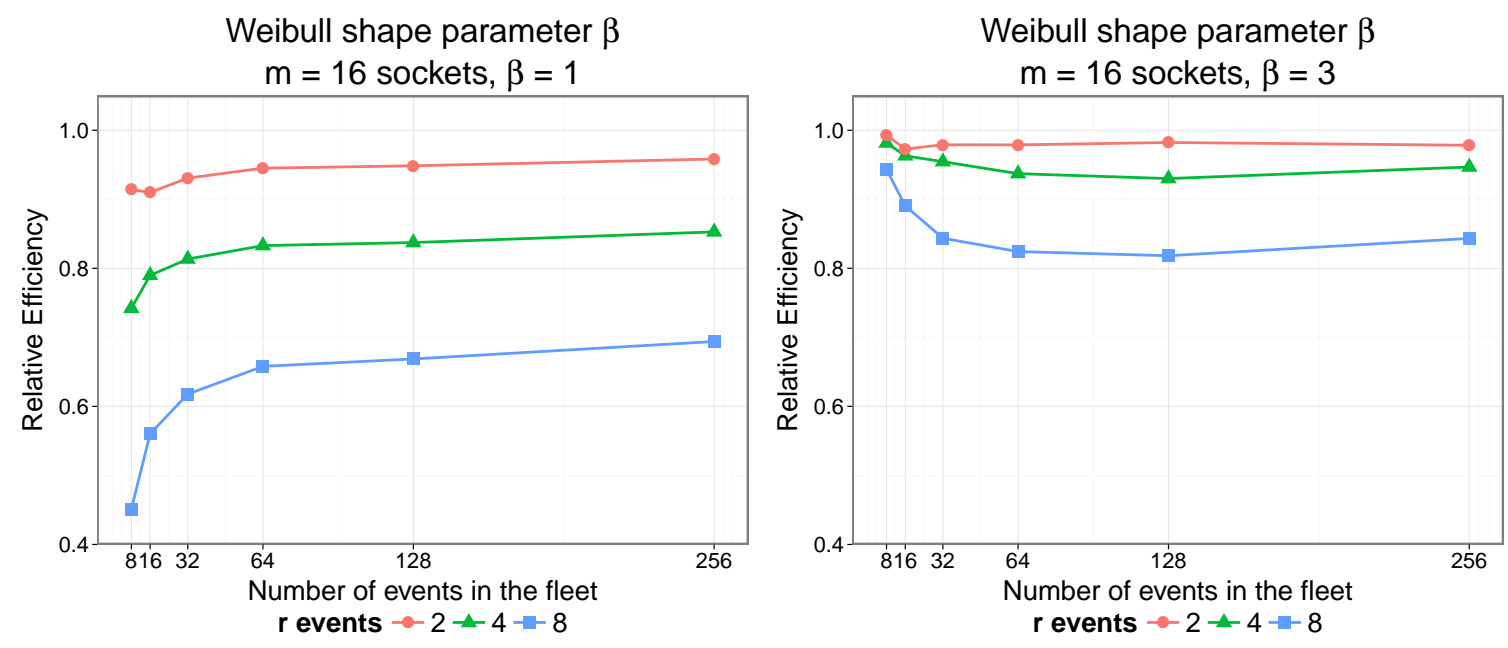

Quantile $t_{0.1}$ $\mathrm{m}=16$ sockets, $\beta=1$

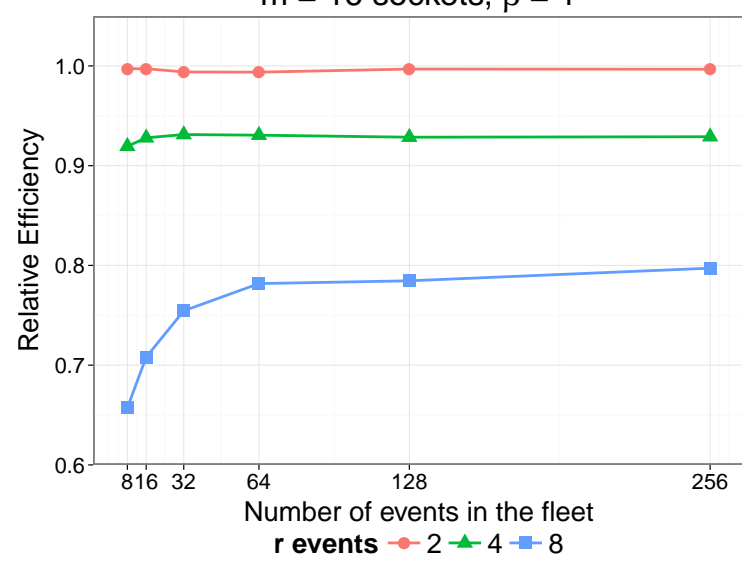

Quantile $t_{0.1}$ $m=16$, sockets, $\beta=3$

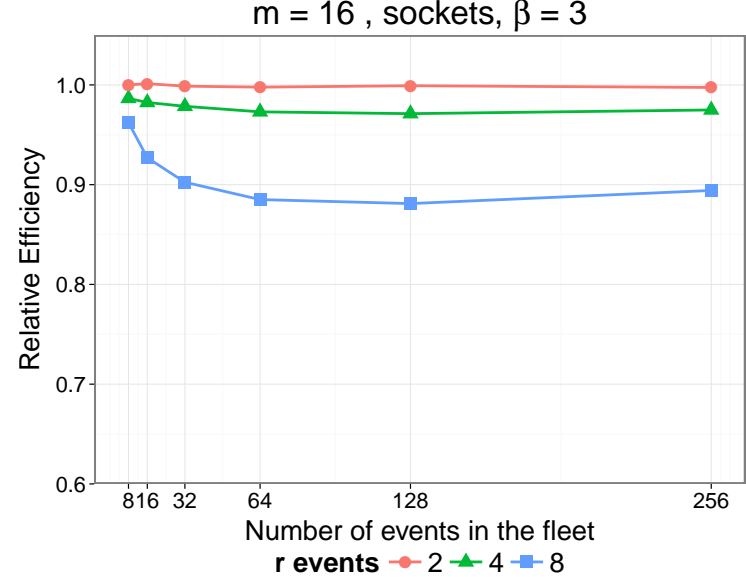

Figure 3: Relative efficiency curves of the idML estimator for estimating $\beta$ and $t_{0.1}$ at different values of the number of events $r$, keeping the number of sockets $m$ fixed at 16 .

As shown in Figure 3 , the patterns of the RE curves are similar for estimating $\beta$ and $t_{0.1}$. From these plots we see the convergence behavior of the RE curves of the idML estimator. The RE changes (increases when $\beta=1$ or decreases when $\beta=3$ ) as the number of events in the fleet increases (e.g., from 8 to 64 ). Then the RE gradually converges to a limiting value as the number of events in the fleet increases up to 256. Regarding the $\beta$ effect, Figure 3 suggests that when $\beta=3$, the idML estimator has a $\mathrm{RE}$ that is relatively higher than that when $\beta=1$.

The RE for $r=2$ is consistently larger than the other two values of $r$, having a limiting value around 0.95 for estimating $\beta$ when $\beta=1$ and closer to 1 in the other three cases. The idML estimator corresponding to $r=8$ has the lowest RE. For the three values of $r$, the fraction of sockets having events $(r / m)$ are $0.125,0.25$, and 0.5 , respectively. Therefore keeping the number of socket $m$ fixed, the idML estimator tends to have a high efficiency when there are fewer events within one system. 

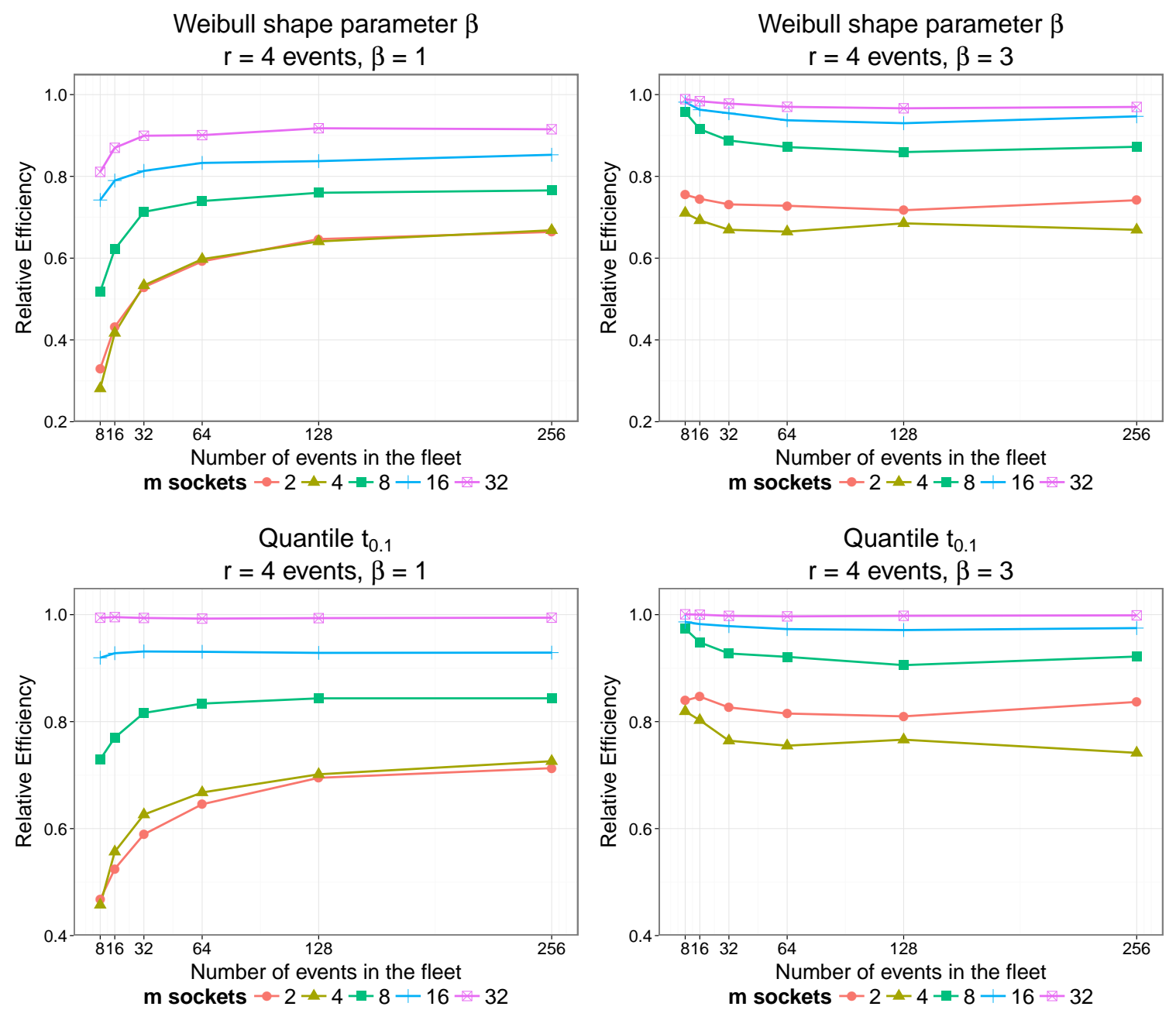

Figure 4: Relative efficiency curves of the idML estimator for estimating $\beta$ (top) and $t_{0.1}$ (bottom) for different values of the number of sockets $m$, keeping number of events per system fixed at $r=4$.

\section{The number of sockets $(m)$ effect}

Secondly, we investigate the effect of the number of sockets $m$ on the RE of the idML estimator, keeping the number of events $r$ within each system fixed. Figure 4 shows a set of relative efficiency curves for five different values of $m(2,4,8,16$, and 32) and two different values of $\beta$ (1 and 3), assuming the number of events $r=4$ within each system. Similar to Figure 3 , the top-left and top-right plots show the RE curves for estimating the Weibull shape parameter $\beta$, corresponding to $\beta=1$ and $\beta=3$, respectively. The bottom two plots are the RE plots for estimating the quantile $t_{0.1}$. From these plots, we see that the idML estimator tends to have higher RE when there is a larger number of sockets $m$ within the SRP. The curve corresponding to $m=32$ is always on top (with a limiting value near 1 ), followed by the curves, which corresponding to $m=16$ and $m=8$. The RE curves for $m=2$ and $m=4$ have similar performance when $\beta=1$, and they have relatively lower RE compared to those with a larger number of sockets $m$. 


\subsection{Confidence interval performance}

For a parameter of interest (e.g., Weibull shape parameter $\beta$ or a particular quantile $t_{p}$ ), let $100(1-\alpha) \%$ be the nominal coverage probability of the confidence interval procedure and let $100(1-\widehat{\alpha}) \%$ denote the Monte Carlo estimate from the 5,000 simulated data sets, where $\widehat{\alpha}$ is the proportion of intervals which do not contain the true value of the parameter. In our simulation, we studied the coverage probabilities of one-sided lower and upper $100(1-\alpha / 2) \%$ confidence intervals for both LR and Wald interval procedures. Then the two-sided $100(1-\alpha) \%$ confidence intervals can be obtained by combining the lower and upper $100(1-\alpha / 2) \%$ confidence intervals. The level of $\alpha$ used in our simulation is 0.05 .
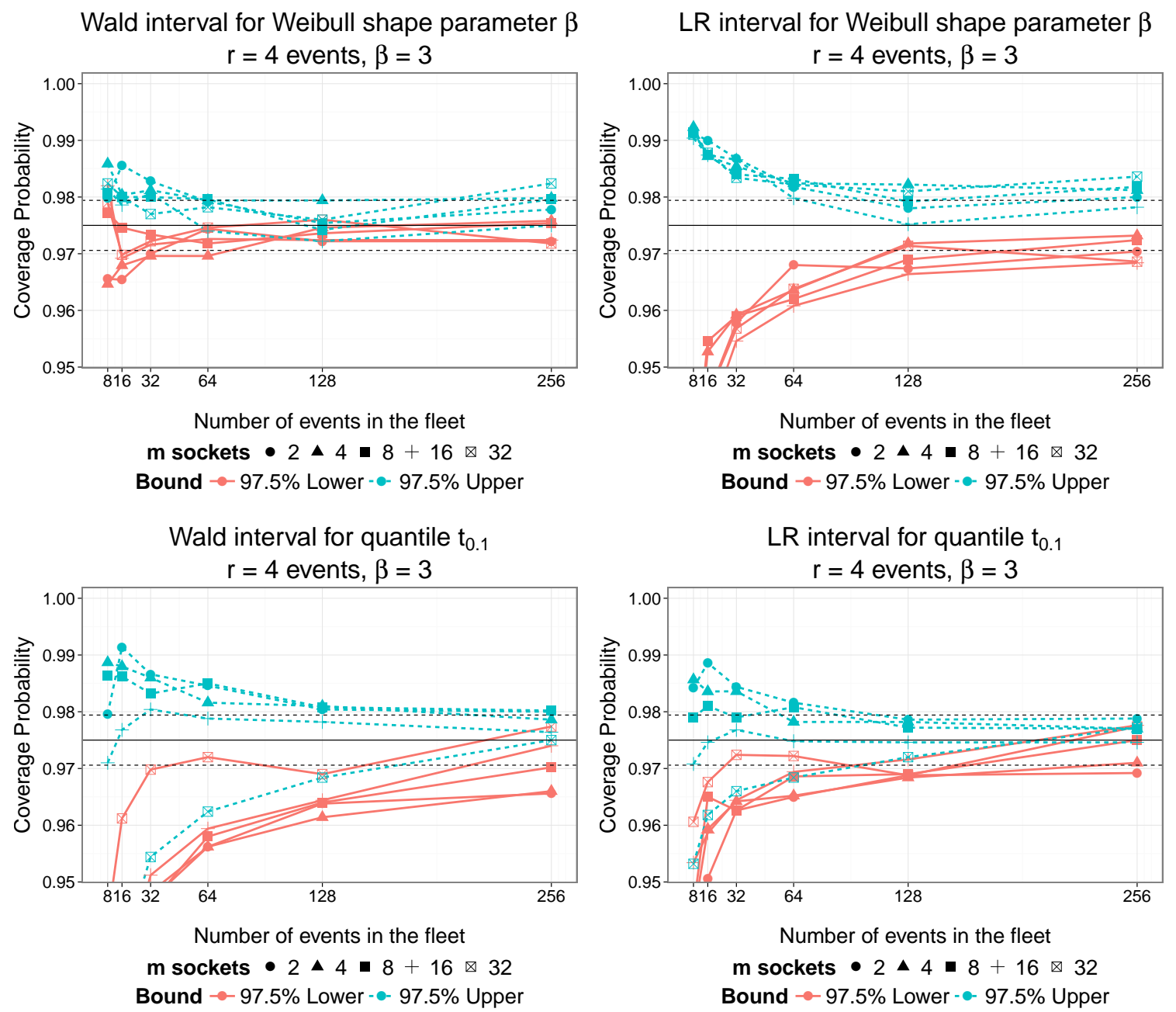

Figure 5: Coverage probability for the Weibull shape parameter $\beta$ (top) and quantile $t_{0.1}$ (bottom) of $97.5 \%$ one-sided Wald CI (left) and LR CI (right), keeping the number of events $r$ fixed at 4 .

Figure 5 shows a set of plots showing the coverage probability curves of one-sided lower $97.5 \%$ and upper $97.5 \%$ confidence intervals for estimating the Weibull shape parameter $\beta$ (the first row) and the quantile $t_{0.1}$ (the second row), using the Wald interval procedure (the first column) and the LR interval procedure (the second column). For all of the four plots, the data were 
simulated from a Weibull distribution with $\eta=1$ and $\beta=3$ and there are $r=4$ events in each SRP (the results for the situation where $\beta=1$ are similar to those for $\beta=3$ and are not shown here). In each plot, the solid curves and the dashed curves correspond to the onesided $97.5 \%$ lower bounds and $97.5 \%$ upper bounds, respectively. Different symbols are used to represent the number of sockets $m$. The standard error of the coverage probability for onesided $97.5 \%$ confidence bounds is $[0.025(1-0.025) / 5000]^{1 / 2}=0.0022$ from 5,000 simulations. The two dashed horizontal lines in the plots highlight the region containing the values within two standard errors of the nominal coverage $97.5 \%$. Using a similar scenario, Figure 6 shows the coverage probability curves corresponding to different values of $r$ for one-sided confidence intervals, keeping the number of sockets $m$ fixed at 16 .
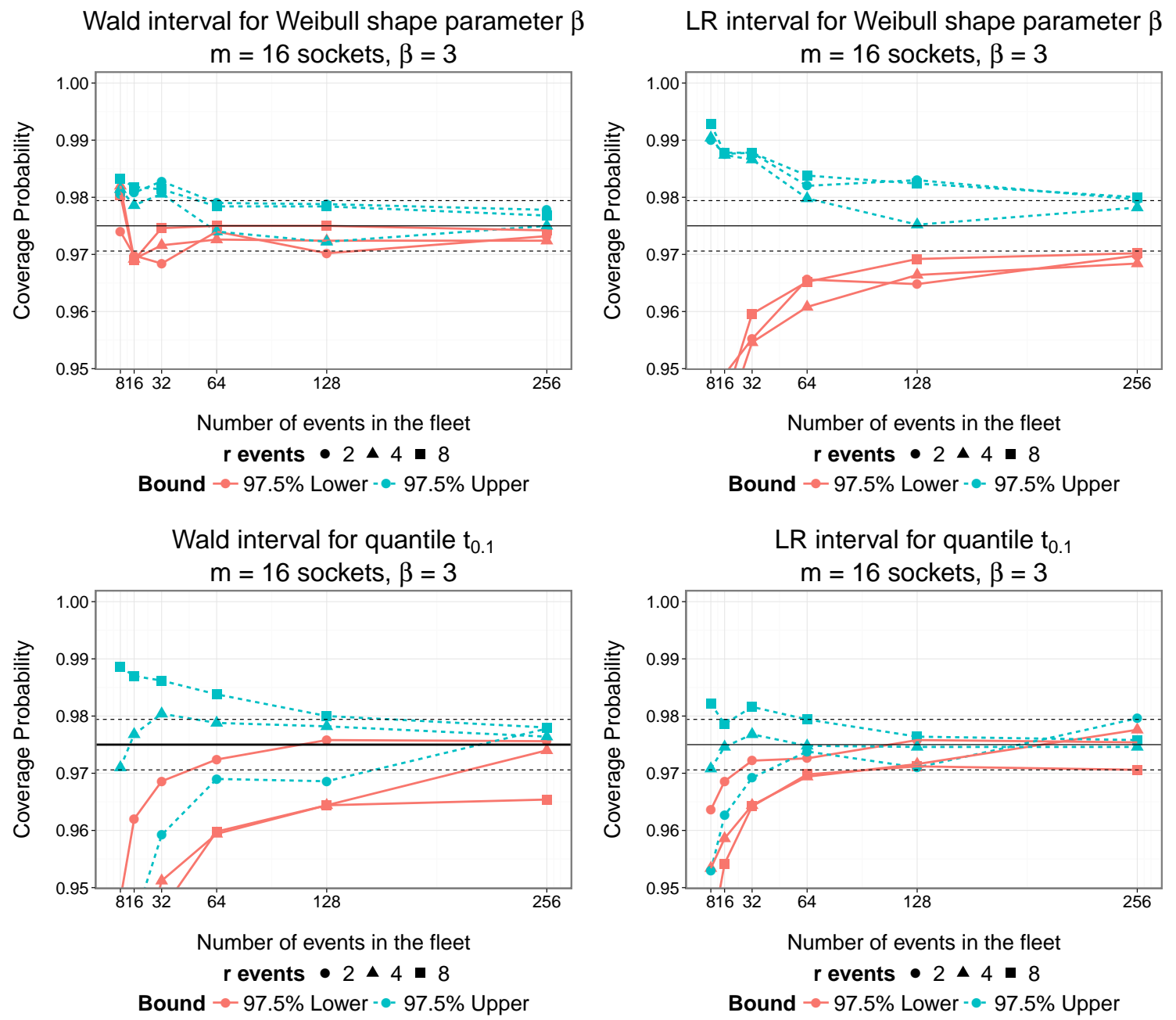

Figure 6: Coverage probability for the Weibull shape parameter $\beta$ (top) and quantile $t_{0.1}$ (bottom) of $97.5 \%$ one-sided Wald CI (left) and LR CI (right), keeping the number of sockets $m$ fixed at 16 .

From Figures 5 and 6, we see that even when there are large number of events (e.g., 256) in the fleet, the convergence of the coverage probability to the nominal coverage is not exact. In Section 1.2, we have seen the potential bias of the idML estimator at different combinations of levels for $m$ and $r$. In our simulation, we increased the number of events in the fleet by increasing 
the number of systems, while keeping the number of events $r$ within each system constant. Thus the bias of the idML estimator will not go to zero as the number of systems in the fleet increases and the limiting coverage probability may not be exactly equal to the nominal confidence level.

\section{Partition Probabilities}

To better understand when the idML estimators are relatively efficient, we conducted a small simulation study to obtain insight about the partition probabilities. As described in the paper, for a system with $r$ events and $m$ sockets, there are $h$ different partitions: $\left(\mathcal{E}_{1}^{r}, \mathcal{E}_{2}^{r}, \ldots, \mathcal{E}_{h}^{r}\right)$. For the $i$ th partition $\mathcal{E}_{i}^{r}=\left(r_{1}, r_{2}, \ldots, r_{l_{i}}\right)$, where $i=1,2, \ldots, h, l_{i} \leq m$, there is a probability $p_{i}$ that an SRP with $r$ events has a partition that is equal to partition $\mathcal{E}_{i}^{r}$, where $0 \leq p_{i} \leq 1$ and $\sum_{i=1}^{h} p_{i}=1$.

Suppose one system has an event history $\mathcal{H}_{\tau_{c}}=\left(\tau_{1}, \tau_{2}, \ldots, \tau_{r}, \tau_{c}\right)$. Then the likelihood of the SRP can be viewed as a sum of the likelihoods corresponding to each partition of the $r$ events. That is,

$$
L\left(\boldsymbol{\theta} ; \mathcal{H}_{\tau_{c}}\right)=\sum_{i=1}^{h} k_{i} \sum_{j=1}^{s_{i}} L_{i, j}\left(\boldsymbol{\theta} ; \mathcal{H}_{\tau_{c}}\right)=\sum_{i=1}^{h} L_{i}\left(\boldsymbol{\theta} ; \mathcal{H}_{\tau_{c}}\right)
$$

where $L_{i}\left(\boldsymbol{\theta} ; \mathcal{H}_{\tau_{c}}\right)=k_{i} \sum_{j=1}^{s_{i}} L_{i, j}\left(\boldsymbol{\theta} ; \mathcal{H}_{\tau_{c}}\right)$ is the likelihood for partition $\mathcal{E}_{i}^{r}$.

Let $\widehat{\boldsymbol{\theta}}$ denote the idML estimate of $\boldsymbol{\theta}$, then an estimate of probability for partition $i$ is

$$
\widehat{p}_{i}=\frac{L_{i}\left(\widehat{\boldsymbol{\theta}} ; \mathcal{H}_{\tau_{c}}\right)}{\sum_{i=1}^{h} L_{i}\left(\widehat{\boldsymbol{\theta}} ; \mathcal{H}_{\tau_{c}}\right)}=\frac{k_{i} \sum_{j=1}^{s_{i}} L_{i, j}\left(\widehat{\boldsymbol{\theta}} ; \mathcal{H}_{\tau_{c}}\right)}{\sum_{i=1}^{h} k_{i} \sum_{j=1}^{s_{i}} L_{i, j}\left(\widehat{\boldsymbol{\theta}} ; \mathcal{H}_{\tau_{c}}\right)}
$$

To illustrate this, for $r=4$ events, there are $h=5$ possible partitions, as shown in Table 1 . The first partition $\mathcal{E}_{1}^{4}=(4)$ indicates that all the four events occurred in one socket. In other words, there is only one event-containing socket among all the $m$ sockets. For the fifth partition $\mathcal{E}_{5}^{4}=(1,1,1,1)$, there are four event-containing sockets with one event per socket. If the number of sockets $m$ is less than 4 , then the partition $\mathcal{E}_{5}^{4}=(1,1,1,1)$ can't occur.

Table 1: Five partitions for $r=4$ events

\begin{tabular}{cc}
\hline$i$ & Partitions $\mathcal{E}_{i}^{4}$ of $r=4$ \\
\hline 1 & $(4)$ \\
2 & $(3,1)$ \\
3 & $(2,2)$ \\
4 & $(2,1,1)$ \\
5 & $(1,1,1,1)$ \\
\hline
\end{tabular}


To describe what the estimates of the probabilities for these partitions look like, we simulated six data sets with different combinations of number of sockets $m(2,4$, and 32$)$ and Weibull shape parameter $\beta$ (1 and 3). Each data set consists of two SRPs, and there are $r=4$ events within each SRP. For each simulated data set, we obtained the idML estimate $\widehat{\boldsymbol{\theta}}$ and calculated the estimate of probability $\widehat{p}_{i}$ for each partition within each SRP according to (2). Bar charts are used to present these estimates $\widehat{p}_{i}$ of the probabilities for the partitions in Figure 7. The plots in the first row and second row correspond to the data sets simulated from Weibull distribution with $\beta=1$ and $\beta=3$, respectively. The three pairs of plots within each row correspond to three simulated data sets with number of sockets $m$ per SRP equal to 2,4 , and 32 , respectively.
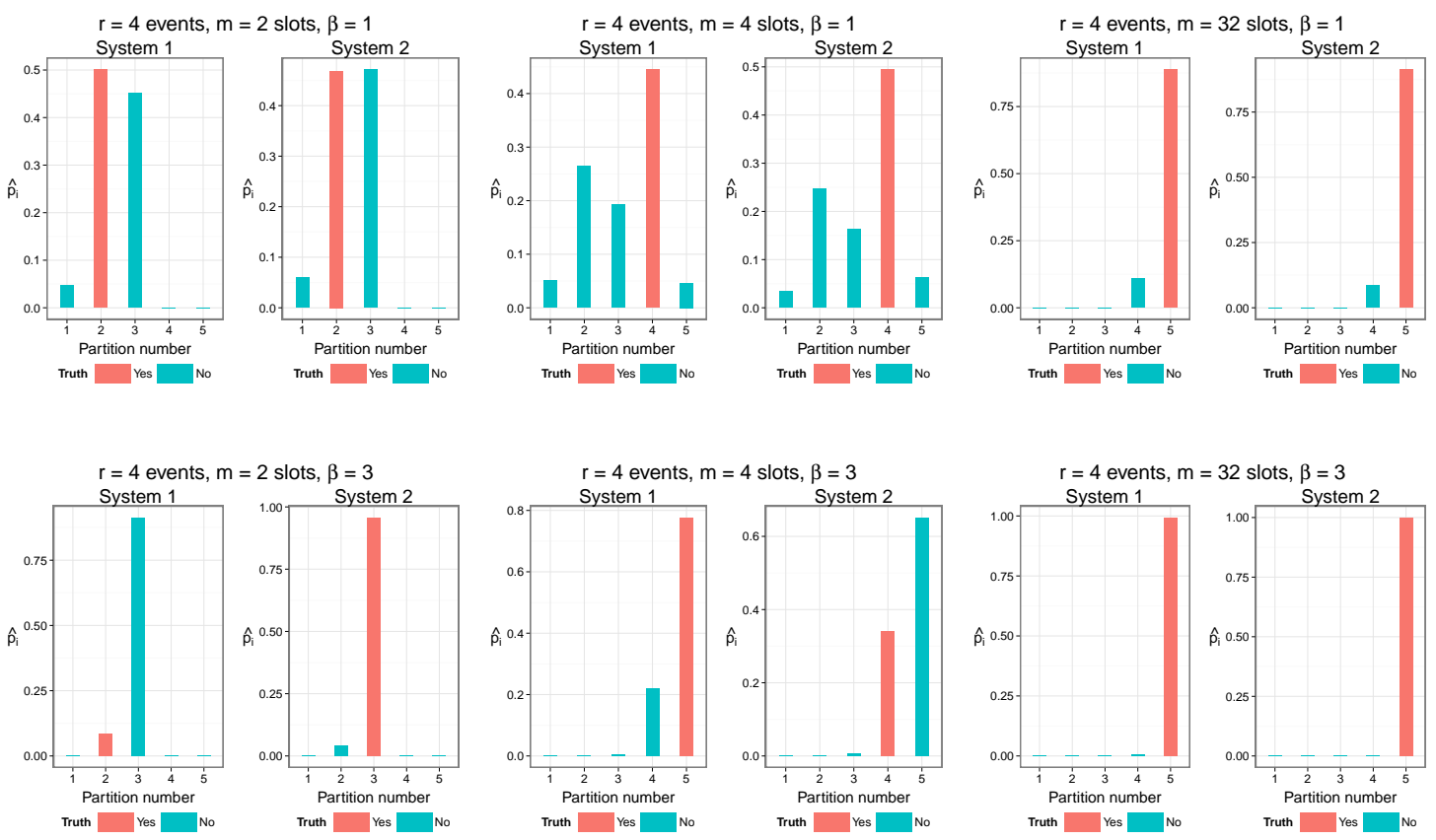

Figure 7: Estimates of probability for the five partitions shown in Table 1, for the six simulated data sets for different combinations of $m(2,4$, and 32$)$ and $\beta$ (1 and 3).

\section{The number of sockets $m$ effect}

The top-left plot refers to a data set which was simulated from a Weibull $(1,1)$ distribution. In this data set, there are $n=2$ independent SRPs, and each SRP has $r=4$ events and $m=2$ sockets. Thus the fraction of sockets with events is $r / m=2 / 4=0.5$. Within each SRP, the probability estimates $\widehat{p}_{i}$ for the five partitions shown in Table 1 are presented using vertical bars whose height is proportional to the value of $\widehat{p}_{i}$. The red bar indicates the true partition that actually happened behind the simulation. Since there are only $m=2$ sockets within each SRP, partitions $\mathcal{E}_{4}^{4}=(2,1,1)$ and $\mathcal{E}_{5}^{4}=(1,1,1,1)$ in Table 1 can't occur. Thus the values of $\widehat{p}_{i}$ are zero for the last two partitions. According to the plot, the second partition $\mathcal{E}_{2}^{4}=(3,1)$ and the third partition $\mathcal{E}_{3}^{4}=(2,2)$ have higher probability (both are around 0.4 to 0.5 ) than the first partition $\mathcal{E}_{1}^{4}=(4)$ (with a probability $\approx 0.05$ ), which means that the $r=4$ events are more likely to spread out across the sockets (i.e., take place in different sockets), given the event history of the data set and this particular combination of factors where $m=2$ and $\beta=1$. For these simulated data, the second partition $\mathcal{E}_{2}^{4}=(3,1)$ is the known true partition for both SRPs. But 
our proposed idML estimation gives approximately equal values for $\widehat{p}_{2}$ and $\widehat{p}_{3}$, which suggests that there is about 50 percent chance to provide a correct guess for the true partition. This explains why in Figure 4 the RE curve corresponding to $m=2$ tends to be lower than the other RE curves, especially for $m=8,16$, and 32 sockets.

The second plot in the first row shows the case which is similar to the one corresponding to the top-left plot, except that the number of sockets within each SRP is $m=4$. In this case all the five different partitions are possible, and the fraction of sockets with events is $r / m=4 / 4=1$. Note that the true partition is $\mathcal{E}_{4}^{4}=(2,1,1)$ for both of the simulated SRPs. The idML estimation provides positive estimates of the probabilities for all the partitions, with the forth partition $\mathcal{E}_{4}^{4}=(2,1,1)$ having the highest probability $(\approx 0.5)$. This is consistent with what we have observed in Figure 4 that the RE curves for $m=2$ and $m=4$ have similar performances.

When the number of sockets $m$ increases to 32 (corresponding to the top-right plot in Figure 7), the fraction of sockets with events becomes $r / m=4 / 32=0.125$. In this case, the probability of the fifth partition $\mathcal{E}_{5}^{4}=(1,1,1,1,1)$ is around 0.8 , which is much larger than that for the other four partitions because this partition is much more likely to take place. Therefore the idML estimator has a high relative efficiency when $m=32$, because there is a higher probability to provide relatively more information about which partition actually occurred.

For the cases where the data sets are simulated from a Weibull distribution with $\beta=3$ (the three plots in the second row of Figure 7), we see that the idML estimation tends to put the majority of probability on a few partitions, which is due to the smaller variability in the simulated data. For $m=2$ (the bottom-left plot), both of the SRPs give an estimate of probability around 0.9 for the partition $\mathcal{E}_{3}^{4}=(2,2)$, which suggests that this particular partition is more likely to take place using the idML estimation. The true partitions, however, are $\mathcal{E}_{2}^{4}=(3,1)$ and $\mathcal{E}_{3}^{4}=(2,2)$ for the two SRPs respectively. For $m=4$, the idML procedure suggests that the partition $\mathcal{E}_{5}^{4}=(1,1,1,1,1)$ (with a probability between 0.7 and 0.8 ) and $\mathcal{E}_{4}^{4}=(2,1,1,1$ ) (with a probability between 0.2 and 0.3 ) are most likely to happen, while the other three partitions have probability close to zero. When there are $m=32$ sockets in each SRP (the bottom-right plot), the true partition $\mathcal{E}_{5}^{4}=(1,1,1,1,1)$ has a probability approximately equal to 1 , which means that the $r=4$ events tend to take place in four different sockets and it is very unlikely that there is one socket that has more than one event. Thus for smaller values of $r / m$, the RE of the idML estimator tends to be large because only the partition $\mathcal{E}_{5}^{4}=(1,1,1,1,1)$ has high probability.

\section{The number of events per system $r$ effect}

To study the effect of $r$ on the probability of partitions, we simulated two data sets from a Weibull distribution with $\beta=1$. The first one had two systems and $r=4$ events within each system and the other had one SRP with $r=8$ events. For the two simulated data sets, there are $m=16$ sockets in each system and $r \times n=8$ events in the fleet. The top-left plot in Figure 8 presents the estimates $\widehat{p}_{i}$ of the probabilities for the partitions of $r=4$ when $\beta=1$. The proportion of sockets that have events is $r / m=4 / 16=0.25$. The high probability of $\mathcal{E}_{5}^{4}=(1,1,1,1,1)$ indicates that the four events are more likely to take place in four different sockets. For the case where there are $r=8$ events within one system, as shown in the top-right plot of Figure 8, the simulation is more complicated because there are $h=22$ possible partitions (see Table 2). In this data set, $r / m=8 / 16=0.5$ and events will occur in no more than one half

of the sockets. The bar chart shows that the partitions $\mathcal{E}_{16}^{8}=(3,2,1,1,1), \mathcal{E}_{17}^{8}=(3,1,1,1,1,1)$, 
$\mathcal{E}_{19}^{8}=(2,2,2,1,1), \mathcal{E}_{20}^{8}=(2,2,1,1,1,1), \mathcal{E}_{21}^{8}=(2,1,1,1,1,1,1)$ and $\mathcal{E}_{22}^{8}=(1,1,1,1,1,1,1,1)$ all have somewhat larger chance to happen, and $\mathcal{E}_{21}^{8}=(2,1,1,1,1,1,1)$ has highest probability that is a little less than 0.4 . The partition that actually happened was $\mathcal{E}_{20}^{8}=(2,2,1,1,1,1)$.

Table 2: 22 partitions for $r=8$ events

\begin{tabular}{cc}
\hline$i$ & Partitions $\mathcal{E}_{i}^{8}$ of $r=8$ \\
\hline 1 & $(8)$ \\
2 & $(7,1)$ \\
3 & $(6,2)$ \\
4 & $(6,1,1)$ \\
5 & $(5,3)$ \\
6 & $(5,2,1)$ \\
7 & $(5,1,1,1)$ \\
8 & $(4,4)$ \\
9 & $(4,3,1)$ \\
10 & $(4,2,2)$ \\
11 & $(4,2,1,1)$ \\
12 & $(4,1,1,1,1)$ \\
13 & $(3,3,2)$ \\
14 & $(3,3,1,1)$ \\
15 & $(3,2,2,1)$ \\
16 & $(3,2,1,1,1)$ \\
17 & $(3,1,1,1,1,1)$ \\
18 & $(2,2,2,2)$ \\
19 & $(2,2,2,1,1)$ \\
20 & $(2,2,1,1,1,1)$ \\
21 & $(2,1,1,1,1,1,1)$ \\
22 & $(1,1,1,1,1,1,1,1)$ \\
\hline
\end{tabular}

We also simulated two similar data sets from a Weibull distribution with $\beta=3$. According to the bottom-left and bottom-right plots in Figure 8, the idML estimation gives a relatively higher probability for the true partition as compared to the case when $\beta=1$, therefore resulting in higher values of $\mathrm{RE}$ for the idML estimator. The probability for partition $\mathcal{E}_{5}^{4}=(1,1,1,1)$ is almost 1 for $r=4$ and $m=16$ (the bottom-left plot), suggesting that it is the dominant partition under this scenario. The bottom-right plot suggests that for $r=8$, the highest probability is only 0.5 among all the possible partitions, and the partition $\mathcal{E}_{21}^{8}=(2,1,1,1,1,1,1)$ (the true partition actually occurred) has a probability 0.4 . Thus when there are $r=8$ events in one system, the chance to provide a correct guess for the true partition is much smaller than the case when $r=4$. This explains why the RE curve for $r=4$ is higher than the one for $r=8$ in Figure 3 , 

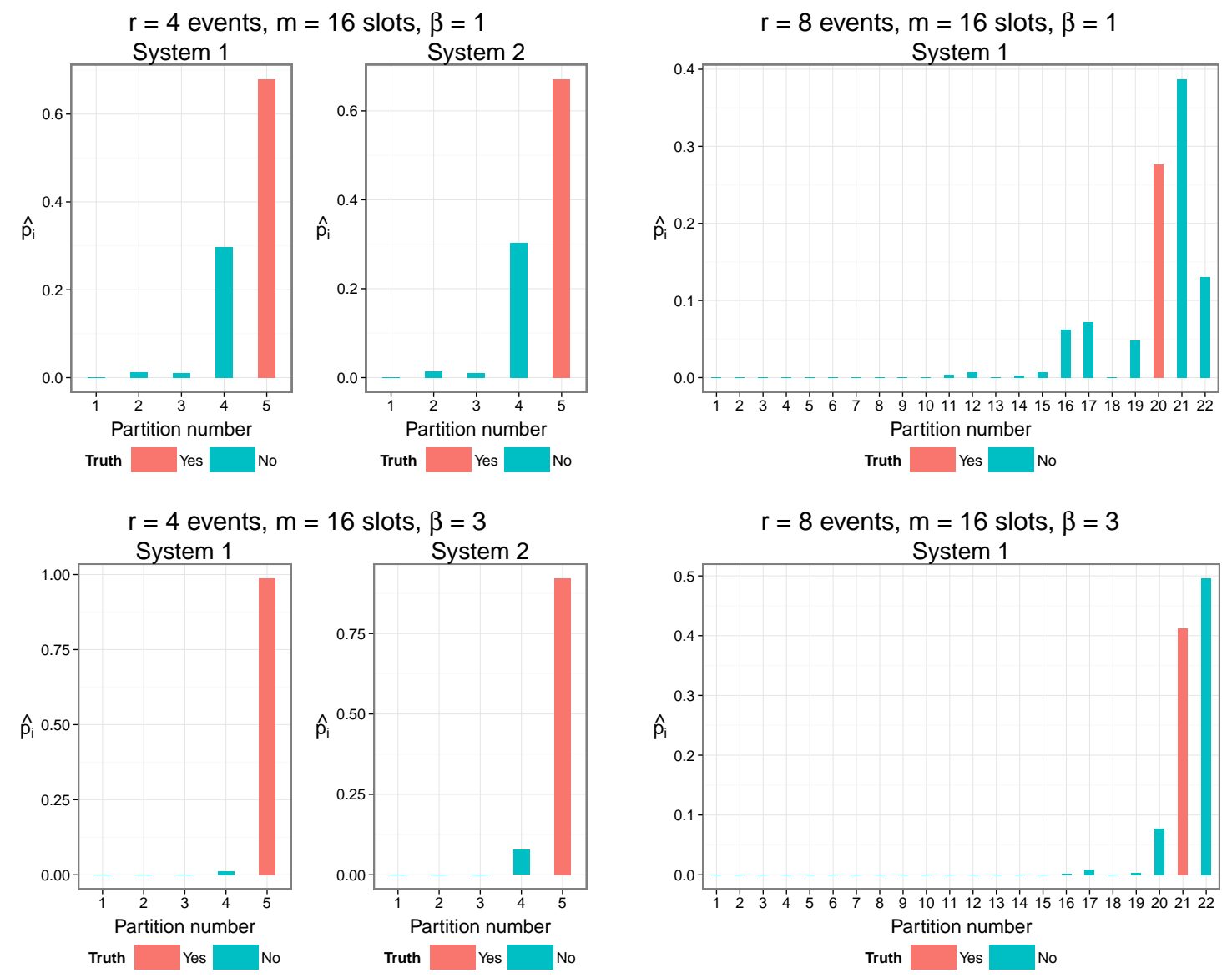

Figure 8: Estimates of probability for the partitions of $r=4$ (shown in Table 1), and partitions of $\mathrm{f} r=8$ (shown in Table 2), for the simulated data sets at four different combinations of $r$ (4 and 8 ) and $\beta$ (1 and 3). 